\title{
Erratum to: Laparoscopic versus robot-assisted bilateral nerve-sparing radical prostatectomy: comparison of pentafecta rates for a single surgeon
}

\author{
Anastasios D. Asimakopoulos $\cdot$ Roberto Miano • \\ Nicola Di Lorenzo $\cdot$ Enrico Spera • \\ Giuseppe Vespasiani · Camille Mugnier
}

Published online: 31 August 2013

(C) Springer Science+Business Media New York 2013

\section{Erratum to: Surg Endosc}

DOI 10.1007/s00464-013-3046-9

In the Results section of the Abstract, the phrase:

"Of the 140 patients who failed pentafecta, 90 (64.3\%) missed a single parameter, and the difference between the groups was significant (80\% LRP vs $53.3 \%$ RALP, $p=0.007)$."

Should be changed as follows:

"Of the 140 patients who failed pentafecta, 90 (64.3\%) missed a single parameter. In these cases, erectile deficit was the leading cause of pentafecta failure, with a significant difference between groups (80\% LRP cases that missed potency recovery vs $53.3 \%$ RALP, $p=0.007)$."

The online version of the original article can be found under doi:10.1007/s00464-013-3046-9.

A. D. Asimakopoulos $(\bowtie) \cdot$ E. Spera

UOC of Urology, Department of Surgery, University of Tor

Vergata, Policlinico Casilino, Rome, Italy

e-mail: tasospao2003@yahoo.com

\author{
A. D. Asimakopoulos - C. Mugnier \\ Division of Urology, Clinique Saint-Augustin, Bordeaux, France \\ R. Miano - G. Vespasiani \\ UOC of Urology, Department of Surgery, University of Tor \\ Vergata, Policlinico Tor Vergata, Rome, Italy \\ N. Di Lorenzo \\ Department of Surgical Sciences, University of Tor Vergata, \\ Policlinico Tor Vergata, Rome, Italy
}

